\title{
In vitro regulation of local secretion and contraction of the bovine oviduct: stimulation by luteinizing hormone, endothelin- 1 and prostaglandins, and inhibition by oxytocin
}

\author{
M P B Wijayagunawardane, A Miyamoto ${ }^{1}$, Y Taquahashi ${ }^{2}$, \\ C Gabler ${ }^{3}$, T J Acosta, M Nishimura ${ }^{2}$, G Killian ${ }^{3}$ and K Sato \\ Department of Theriogenology, Obihiro University of Agriculture and Veterinary Medicine, Obihiro 080-8555, Japan \\ ${ }^{1}$ Department of Animal Science, Obihiro University of Agriculture and Veterinary Medicine, Obihiro 080-8555, Japan \\ ${ }^{2}$ Department of Veterinary Pharmacology, Obihiro University of Agriculture and Veterinary Medicine, Obihiro 080-8555, Japan \\ ${ }^{3}$ J O Almquist Research Center, The Pennsylvania State University, University Park, Pennsylvania 16802, USA \\ (Requests for offprints should be addressed to A Miyamoto, Department of Animal Science, Obihiro University of Agriculture and Veterinary Medicine, \\ Obihiro 080-8555, Japan; Email: akiomiya@obihiro.ac.jp) \\ (M P B Wijayagunawardane is now at Department of Animal Science, University of Peradeniya, Peradeniya, Sri Lanka)
}

\begin{abstract}
The precise regulatory mechanisms of cyclic oviductal contraction in the cow are unclear. The purpose of this study was to evaluate the effect of luteinizing hormone $(\mathrm{LH})$, steroids, prostaglandins (PGs) and peptides on the oviductal contraction and secretion of PGs and endothelin (ET-1). In addition, the cyclic expression of mRNA for ET-1 and its receptors (ET-R) was evaluated by reverse transcription-polymerase chain reaction (RT-PCR). In the in vitro microdialysis study, an infusion of $\mathrm{LH}$ alone or in combination with progesterone $\left(\mathrm{P}_{4}\right)$, estradiol-17 $\beta\left(\mathrm{E}_{2}\right)$ and/or ET-1 stimulated pronounced release of $\mathrm{PGE}_{2}$, $\mathrm{PGF}_{2 \alpha}$ and ET-1 in the oviducts from cows in the follicular and postovulatory phases. The addition of $\mathrm{LH}$, $\mathrm{LH}+\mathrm{P}_{4}+\mathrm{E}_{2}$ and/or ET-1 to the medium increased the amplitude of oviductal contraction. However, oxytocin (OT) completely blocked the responses of oviductal
\end{abstract}

secretion and contraction. In contrast, these substances did not show any effect in the oviducts from cows in the mid luteal phase. Similar expression patterns of mRNA encoding for ET-R type A and type B were found, which were highest during the postovulatory phase, lower during the luteal phase, with the lowest expression during the follicular phase. We suggest that the preovulatory LH surge, together with increasing $\mathrm{E}_{2}$ levels from the Graafian follicle and a basal $\mathrm{P}_{4}$ from regressing corpora lutea (CL), stimulates maximum oviductal production of $\mathrm{PG}$ and ET-1, resulting in oviductal contraction for a rapid transport of gametes. OT released from the newly-formed CL may block these mechanisms, and slow contractions for transport of the embryo to the uterus.

Journal of Endocrinology (2001) 168, 117-130

\section{Introduction}

The oviduct plays a central role in the events of fertilisation and early embryonic development by providing an environment that facilitates sperm transport and capacitation, and oocyte transport and maturation. The simultaneous opposite movements of gametes to the fertilization site and the subsequent passage of the embryo into the uterus at the precise developmental stage are essential for successful pregnancy. Different contractile patterns of the oviduct occur during the periovulatory period for the movement of the zygote, which are strictly regulated by endocrine and paracrine/autocrine factors.

Studies on spontaneous oviduct contraction in the cow revealed low amplitude and frequency motility patterns during the luteal phase (Bennett et al. 1988), which gradually increased with the drop in serum progesterone $\left(\mathrm{P}_{4}\right)$ prior to estrus (Ruckebusch \& Bayard 1975). Both amplitude and frequency reach a maximum during estrus, but rapidly diminish over the next 3 days (Ruckebusch \& Bayard 1975, Bennett et al. 1988). The acute withdrawal of endogenous estradiol-17 $\beta\left(\mathrm{E}_{2}\right)$ has been shown to be a potent stimulus to rabbit oviductal smooth muscle and contributes to an increase in muscular activity at ovulation (Halbert \& Conrad 1975). During the retention of ova in porcine oviducts, the active preovulatory contractions disappeared, and ovum transport was arrested at the ampullar-isthmic junction (Rodriguez-Martinez et al. 1982b). In addition, an isthmic sphinctering effect controlling ovum transport through the oviduct has been reported (Lindblom et al. 1979). The motility pattern of sow oviduct during estrus was mostly antiperistaltic, 
presumably related to sperm transport. However, during the periovulatory period the number of peristaltic and antiperistaltic waves became equal, perhaps in relation to the transport of gametes to the fertilization site (Rodriguez-Martinez et al. 1982a). These observations provide evidence that patterns of oviduct contraction during the periovulatory period are temporally and strictly controlled by local mechanisms that are responsive to the needs of the zygote.

The mechanisms involved in the regulation of contractions to facilitate gamete and embryo transport in the bovine oviduct are still unclear. The purpose of this study was, therefore, to examine the in vitro effect of luteinizing hormone (LH), oxytocin (OT) and ovarian steroids on oviduct contractions, as well as the secretion of prostaglandins (PG) and endothelin-1 (ET-1) by bovine oviduct epithelial cells. To investigate the secretions of the oviducts in vitro, we utilized a microdialysis system implanted in the lumen of oviducts that were maintained in organ culture chambers. In addition, the expression of mRNA for ET-1 and ET-1 receptors (ET-R) was evaluated in oviducts recovered during the follicular, postovulatory and luteal phases.

\section{Materials and Methods}

Source of the hormones

$\mathrm{P}_{4}, \mathrm{E}_{2}, \mathrm{PGE}_{2}$ and $\mathrm{PGF}_{2 \alpha}$ were obtained from Sigma Chemical Co. (St Louis, MO, USA). OT and ET-1 were obtained from Peptide Institute Inc. (Osaka, Japan). LH (USDA-bLH-B-6) was a gift from the USDA Animal Hormone program (Baltimore, MD, USA).

\section{Animals and sample collection}

Reproductive tracts were collected from non-pregnant Holstein cows at a local slaughterhouse. The phase of the estrous cycle was determined according to the criteria of Ireland et al. (1980) by visual observation of the ovarian structures and the uterine mucous characteristics. The oviducts were classified as either follicular phase (days 18-20), postovulatory phase with a 3-5 $\mathrm{mm}$ diameter of point of rupture not covered by epithelium (days 1-2), or luteal phase (days 11-17). They were separated from the utero-tubal junction and surrounding connective tissues and trimmed. Oviducts for microdialysis experiments were transported to the laboratory in Medium 199 (M199; $25 \mathrm{mmol} / 1$ Hepes, $0.85 \mathrm{~g} / 1 \mathrm{NaHCO}_{3}, 60 \mathrm{mg} / 1$ penicillin, $100 \mathrm{mg} / 1$ streptomycin, $56 \mathrm{mg} / 1$ ascorbic acid and $2 \mathrm{mg} / 1$ amphotericin B; Sigma Chemical Co.), which was maintained at $38^{\circ} \mathrm{C}$. Oviducts for the contraction experiments were transported in ice-cold Locke Ringer's solution (154 mmol/1 NaCl, 5.6 mmol/1 KCl, $2.4 \mathrm{mmol} / 1 \mathrm{CaCl}_{2}$, $6 \cdot 0 \mathrm{mmol} / 1 \mathrm{NaHCO}_{3}$ and $5 \cdot 6 \mathrm{mmol} / 1$ dextrose, $\mathrm{pH} 7 \cdot 4$ ).
For RNA extraction, the oviducts were frozen immediately in liquid nitrogen, transported to the laboratory and stored at $-80{ }^{\circ} \mathrm{C}$. The ampullary and isthmic regions of oviducts, both ipsilateral and contralateral to the functioning corpora lutea (CL) or developing follicle and ovulation site, were used in this study.

\section{Estimation of the doses of the substances administered in microdialysis and contraction experiments}

The concentrations of different substances used in this study were chosen based on measured concentrations in the bovine oviduct during the normal estrous cycle (Wijayagunawardane et al. 1996, 1998). Diffusion through the microdialysis capillary membrane was estimated as $1 \%$ for steroids and PG, and $0 \cdot 1 \%$ for peptides (Miyamoto \& Schams 1991, Miyamoto et al. 1997). Thus, the doses of steroid and PG were prepared so as to be 100 times greater than the concentrations estimated at the site of action, and the peptides doses were prepared so as to be 1000 times greater.

\section{In vitro microdialysis system of bovine oviduct}

The in vitro microdialysis system of the oviduct was adapted from the method described for the bovine CL (Miyamoto \& Schams 1991). Briefly, the lumen of each $10 \mathrm{~cm}$ long oviductal segment was implanted with a $7 \mathrm{~cm}$ long and $0.2 \mathrm{~mm}$ diameter dialysis capillary membrane (Fresenius SPS 600 Hollow fiber, Fresenius AG, St Wendel, Germany: cut off $40 \mathrm{kDa}$ ) with each end glued to $5 \mathrm{~cm}$ long and $0.3 \mathrm{~mm}$ diameter silastic tubing. Both ends of the oviduct were then fixed to the silastic tubing by Histoacryl Blau (B Braun Melsungen AG, Melsungen, Germany) and incubated in M199 with $0.5 \%$ BSA (Sigma Chemical Co.) in simple organ culture chambers (modified $50 \mathrm{ml}$ Falcon tubes; Becton Dickinson \& Co, Lincoln Park, NJ, USA). In preliminary experiments, differences in response associated with side of ovulation or region of the oviduct were not observed. Therefore, each oviduct was divided into two segments, four oviductal segments from each cow were maintained in one organ culture chamber, and each segment was assigned randomly to different treatments as described below. Medium was continuously exchanged at a flow rate of $50 \mathrm{ml} / \mathrm{h}$ during the whole period of incubation at $38^{\circ} \mathrm{C}$. Both ends of the silastic tubing were connected to Teflon tubing and Ringer's solution was continuously perfused $(1.3 \mathrm{ml} / \mathrm{h})$ from one end using a peristaltic pump, while the other end was connected to a fraction collector. After a 2-h pre-incubation, the perfusate was collected in 4-h fractions for $16 \mathrm{~h}$. The volume of a fraction was approximately $5 \cdot 2 \mathrm{ml}$. Control (Ringer's solution only) and $\mathrm{LH}(1 \mu \mathrm{g} / \mathrm{ml}), \mathrm{P}_{4}(3 \cdot 2 \mu \mathrm{mol} / \mathrm{l}), \mathrm{E}_{2}(370 \mathrm{nmol} / \mathrm{l})$, OT $(100 \mathrm{nmol} / \mathrm{l})$, ET-1 $(100 \mathrm{nmol} / \mathrm{l}), \mathrm{PGE}_{2}(1 \mu \mathrm{mol} / \mathrm{l})$ or $\mathrm{PGF}_{2 \alpha}(100 \mathrm{nmol} / \mathrm{l})$ in Ringer's solution were infused for 

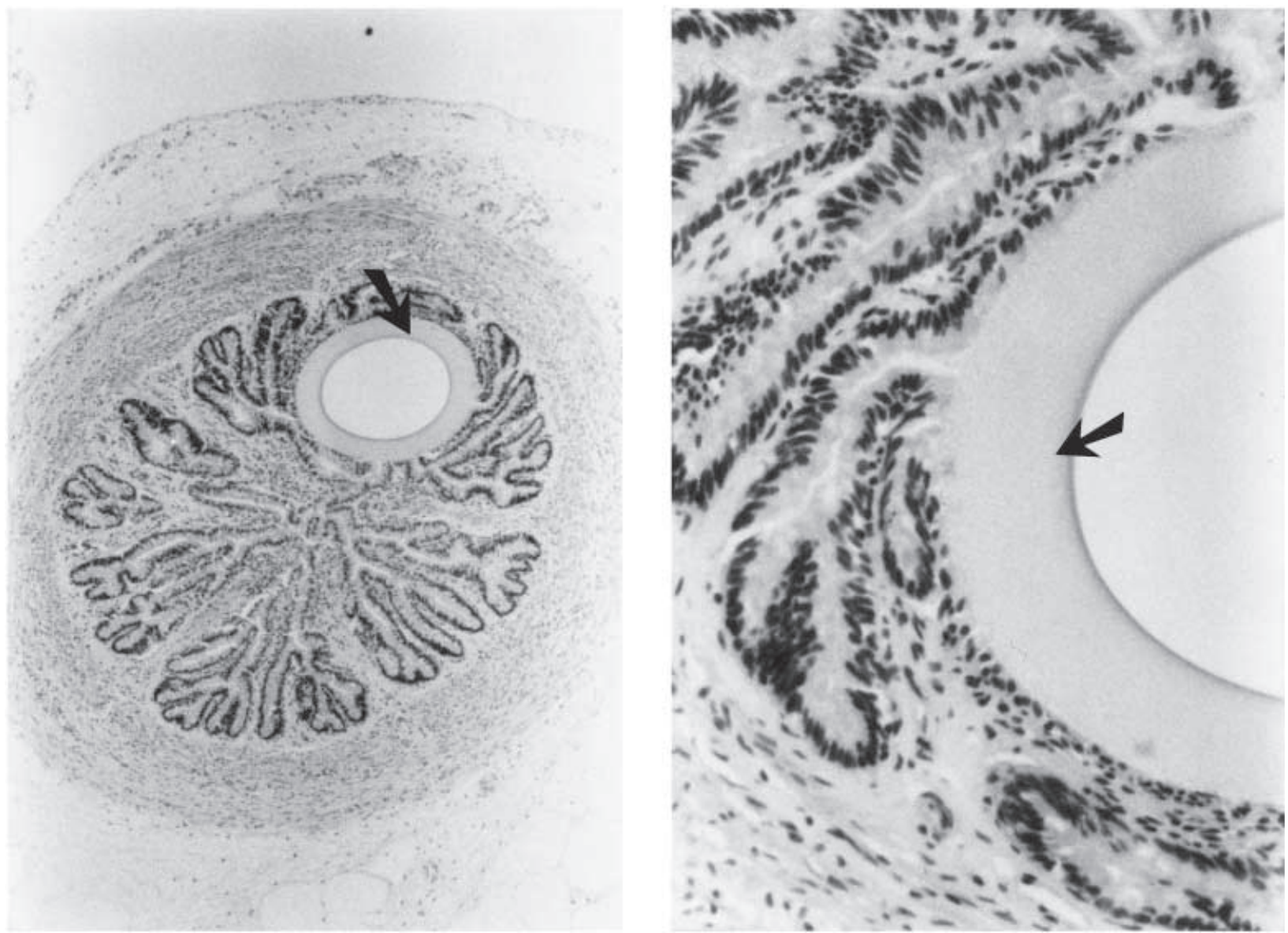

Figure 1 Position of the microdialysis membrane (arrow) in the oviduct. Magnification: left $\times 20$ and right $\times 200$.

$4 \mathrm{~h}$ between 4 and $8 \mathrm{~h}$ of incubation. The same concentrations were also used in the consecutive microdialysis experiments; mixtures of treatment substances (controlRinger's solution only, $\mathrm{P}_{4}+\mathrm{E}_{2}, \mathrm{LH}+\mathrm{E}_{2}, \mathrm{LH}+\mathrm{ET}-1$, $\mathrm{LH}+\mathrm{E}_{2}+\mathrm{P}_{4}, \quad \mathrm{LH}+\mathrm{ET}-1+\mathrm{P}_{4}, \quad \mathrm{LH}+\mathrm{E}_{2}+\mathrm{ET}-1+\mathrm{P}_{4}, \mathrm{OT}+$ $\mathrm{LH}, \mathrm{OT}+\mathrm{LH}+\mathrm{E}_{2}, \mathrm{OT}+\mathrm{E}_{2}+\mathrm{P}_{4}, \mathrm{OT}+\mathrm{E}_{2}+\mathrm{ET}-1$ or $\mathrm{OT}+$ $\mathrm{LH}+\mathrm{ET}-1+\mathrm{E}_{2}$ ) in Ringer's solution were infused for $4 \mathrm{~h}$ between 4 and $8 \mathrm{~h}$ of incubation. The $4-\mathrm{h}$ fractions of perfusate were stored at $-20{ }^{\circ} \mathrm{C}$ until extraction for PG and peptides. After the experiment, the microdialysis capillary membranes implanted in the oviduct lumens were confirmed to be in tight contact with the surface of the epithelial cells (Fig. 1).

\section{Hormone extraction}

The fractions collected were warmed to room temperature, adjusted to $\mathrm{pH} 3.5$ with $1 \mathrm{~mol} / 1 \mathrm{HCl}$ and placed at room temperature for $1 \mathrm{~h}$. PG and ET-1 extractions from microdialysis samples were performed as described earlier (Acosta et al. 1998). As a result of the extractions, PG and ET-1 were concentrated 12- and 52-fold respectively.
Samples were dissolved in assay buffers for enzyme immunoassays (EIAs) for PG (40 mmol/l PBS, $0 \cdot 1 \% \mathrm{BSA}$, $\mathrm{pH} \mathrm{7} \cdot 2)$ and for peptide $\left(42 \mathrm{mmol} / 1 \mathrm{Na}_{2} \mathrm{HPO}_{4}, 8 \mathrm{mmol} / \mathrm{l}\right.$ $\mathrm{KH}_{2} \mathrm{PO}_{4}, 20 \mathrm{mmol} / 1 \mathrm{NaCl}, 4.8 \mathrm{mmol} / 1$ EDTA, 0.05\% BSA, $\mathrm{pH} 7 \cdot 5$ ). The recovery rates, estimated by adding 3 different concentrations of $\mathrm{PGE}_{2}(1,0.5,0.1 \mathrm{ng} / \mathrm{ml})$, $\mathrm{PGF}_{2 \alpha}(1,0 \cdot 5,0 \cdot 1 \mathrm{ng} / \mathrm{ml})$ and ET-1 $(10,5,1 \mathrm{pg} / \mathrm{ml})$ to the Ringer's solution were $78 \%$ for $\mathrm{PGE}_{2}, 75 \%$ for $\mathrm{PGF}_{2 \alpha}$ and $63 \%$ for ET-1. The results presented are corrected for the differences in the recoveries of PG and ET-1.

\section{Measurements of PG and ET-1}

Concentrations of $\mathrm{PGE}_{2}, \mathrm{PGF}_{2 \alpha}$ and ET-1 in Ringer's extracts were measured using second antibody EIAs. The EIA for $\mathrm{PGE}_{2}$ determination was performed as described previously (Wijayagunawardane et al. 1998). The method of EIA for $\mathrm{PGF}_{2 \alpha}$ is described elsewhere (Miyamoto et al. 1995). The ET-1 assay was performed as described earlier (Miyamoto et al. 1997). Within-assay and between-assay coefficients of variance were $7 \cdot 3 \%$ and $11 \cdot 4 \%$ for $\mathrm{PGE}_{2}$, $8 \cdot 2 \%$ and $11 \cdot 8 \%$ for $\mathrm{PGF}_{2 \alpha}$ and $9 \cdot 6 \%$ and $13 \cdot 2 \%$ for ET-1. 
Table 1 Characterization of basal secretion of $\mathrm{PGE}_{2}, \mathrm{PGF}_{2 \alpha}$ and $\mathrm{ET}-1$ in the microdialysis system, and spontaneous contraction of bovine oviducts during different phases of the estrous cycle in vitro

\begin{tabular}{|c|c|c|c|c|c|}
\hline & \multicolumn{3}{|c|}{$\begin{array}{l}\text { Basal secretion } \\
\text { (pmol// Ringer's solution) }\end{array}$} & \multicolumn{2}{|c|}{ Spontaneous contraction } \\
\hline & $\mathrm{PGE}_{2}$ & $\mathrm{PGF}_{2 \alpha}$ & ET-1 & $\begin{array}{l}\text { Amplitude } \\
\text { (gf) }\end{array}$ & $\begin{array}{l}\text { Frequency } \\
\text { (beats/min) }\end{array}$ \\
\hline \multicolumn{6}{|l|}{$\begin{array}{l}\text { Phases of the } \\
\text { estrous cycle }\end{array}$} \\
\hline Follicular & $1515 \pm 596^{a}$ & $1478 \pm 516^{a}$ & $2 \cdot 9 \pm 1 \cdot 3^{a}$ & $0 \cdot 27 \pm 0 \cdot 07^{\mathrm{a}}$ & $3 \cdot 45 \pm 0 \cdot 46^{a}$ \\
\hline Postovulatory & $1784 \pm 437^{a}$ & $1453 \pm 550^{a}$ & $3 \cdot 5 \pm 0 \cdot 5^{\mathrm{a}}$ & $0 \cdot 31 \pm 0 \cdot 06^{\mathrm{a}}$ & $3 \cdot 49 \pm 0 \cdot 46^{a}$ \\
\hline Luteal & $349 \pm 91^{b}$ & $522 \pm 85^{\mathrm{b}}$ & $1 \cdot 4 \pm 0 \cdot 2^{b}$ & $0 \cdot 15 \pm 0 \cdot 09^{b}$ & $1.90 \pm 0.52^{b}$ \\
\hline
\end{tabular}

Means with different superscripts within a column are significantly different $(P<0 \cdot 05)$.

The $\mathrm{ED}_{50} \mathrm{~s}$ for the $\mathrm{PGE}_{2}, \mathrm{PGF}_{2 \alpha}$ and $\mathrm{ET}-1$ assays were $740 \mathrm{pmol} / 1,1000 \mathrm{pmol} / 1$ and $180 \mathrm{pmol} / 1$ respectively, and the ranges of the standard curves for these assays were 85-40 $000 \mathrm{pmol} / 1,20-20000 \mathrm{pmol} / 1$ and 4-4000 $\mathrm{pmol} / 1$ respectively.

\section{Measurement of oviduct contraction}

One end of each of the $10 \mathrm{~mm}$ long oviduct segments was horizontally fixed through the lumen to a plastic holder, and the free end was attached to the arm of a force displacement transducer (TB-625T; Nihon Koden, Tokyo, Japan) with a stainless steel hook and a silk ligature. A basal loading tension of $1 \mathrm{gf}$ was maintained throughout the experiment. The preparation was soaked in Locke Ringer's solution in double-jacketed organ culture chambers (Iwaki Glass Co, Chiba, Japan) and maintained at $36 \pm 0.5{ }^{\circ} \mathrm{C}$. The temperature of the solution in the organ bath was held constant with an external water jacket and a thermoregulatory device (Thermominder Mini-80; Taiyo, Tokyo, Japan), and monitored with a thermistor (Model MAG-II; Shibaura Electric Co, Tokyo, Japan).

Table 2 Effects of the infusion of $\mathrm{P}_{4}, \mathrm{E}_{2}, \mathrm{LH}, \mathrm{ET}-1, \mathrm{OT}, \mathrm{PGE}_{2}$ and $\mathrm{PGF}_{2 \alpha}$ on the release of PGs and ET-1 in microdialyzed cow oviducts from the follicular phase of the estrous cycle $(n=4$, means \pm S.E.M.; control=Ringer's solution only). The data are expressed as percentages of basal secretion $(100 \%$; $0-4 \mathrm{~h})$

\begin{tabular}{|c|c|c|c|c|}
\hline \multirow{2}{*}{ Secretion } & \multirow[b]{2}{*}{ Treatment } & \multicolumn{3}{|c|}{ Incubation period $(\mathrm{h})$} \\
\hline & & $4-8+$ & $8-12$ & $12-16$ \\
\hline $\begin{array}{l}\text { Secretion } \\
\mathrm{PGE}_{2}\end{array}$ & $\begin{array}{l}\text { Control } \\
\mathrm{P}_{4} \\
\mathrm{E}_{2} \\
\text { LH } \\
\text { ET-1 } \\
\text { OT }\end{array}$ & $\begin{array}{l}121 \pm 48 \\
173 \pm 53 \\
142 \pm 33 \\
428 \pm 252 \\
538 \pm 151 \\
232 \pm 125\end{array}$ & $\begin{array}{l}111 \pm 23 \\
139 \pm 38 \\
181 \pm 53 \\
532 \pm 118^{\star *} \\
338 \pm 234 \\
148 \pm 53\end{array}$ & $\begin{array}{l}136 \pm 25 \\
133 \pm 42 \\
214 \pm 54 \\
418 \pm 128^{*} \\
123 \pm 92 \\
134 \pm 49\end{array}$ \\
\hline $\mathrm{PGF}_{2 \alpha}$ & $\begin{array}{l}\text { Control } \\
\mathrm{P}_{4} \\
\mathrm{E}_{2} \\
\text { LH } \\
\text { ET-1 } \\
\text { OT }\end{array}$ & $\begin{array}{l}131 \pm 34 \\
348 \pm 215 \\
732 \pm 123^{* *} \\
638 \pm 158^{* *} \\
748 \pm 138^{* *} \\
123 \pm 48\end{array}$ & $\begin{array}{l}152 \pm 83 \\
435 \pm 142^{*} \\
548 \pm 174^{\star *} \\
734 \pm 257^{*} \\
347 \pm 223 \\
154 \pm 92\end{array}$ & $\begin{array}{l}118 \pm 28 \\
216 \pm 79 \\
635 \pm 248^{*} \\
664 \pm 286 \\
432 \pm 241 \\
221 \pm 98\end{array}$ \\
\hline $\mathrm{ET}-1$ & $\begin{array}{l}\text { Control } \\
\mathrm{P}_{4} \\
\mathrm{E}_{2} \\
\mathrm{LH} \\
\mathrm{OT} \\
\mathrm{PGE}_{2} \\
\mathrm{PGF}_{2 \alpha}\end{array}$ & $\begin{aligned} 121 & \pm 43 \\
154 & \pm 81 \\
138 & \pm 98 \\
519 & \pm 242 \\
98 & \pm 42 \\
118 & \pm 36 \\
127 & \pm 27\end{aligned}$ & $\begin{aligned} 98 & \pm 22 \\
193 & \pm 95 \\
392 & \pm 116^{* *} \\
843 & \pm 98^{* *} \\
115 & \pm 38 \\
128 & \pm 82 \\
154 & \pm 43\end{aligned}$ & $\begin{array}{l}148 \pm 34 \\
188 \pm 67 \\
134 \pm 24 \\
748 \pm 223^{*} \\
147 \pm 45 \\
146 \pm 51 \\
117 \pm 48\end{array}$ \\
\hline
\end{tabular}

Indicates the period of treatments.

${ }^{*} P<0 \cdot 05,{ }^{* *} P<0 \cdot 01$, significantly different from controls. 
Table 3 Effects of combined treatment of hormonal stimulation on the release of PGs and ET-1 in microdialyzed cow oviducts collected from the follicular phase of the estrous cycle $(n=4$, means \pm S.E.M.; control=Ringer's solution only). The data are expressed as percentages of basal secretion $(100 \% ; 0-4 \mathrm{~h})$

\begin{tabular}{|c|c|c|c|c|}
\hline & \multirow[b]{2}{*}{ Treatment } & \multicolumn{3}{|c|}{ Incubation period $(h)$} \\
\hline & & $4-8 \dagger$ & $8-12$ & $12-16$ \\
\hline & Control & $122 \pm 32$ & $101 \pm 28$ & $98 \pm 31$ \\
\hline \multirow{12}{*}{$\mathrm{PGE}_{2}$} & $\mathrm{P}_{4}+\mathrm{E}_{2}$ & $147 \pm 65$ & $274 \pm 78$ & $197 \pm 62$ \\
\hline & $\mathrm{LH}+\mathrm{E}_{2}$ & $458 \pm 113^{*}$ & $724 \pm 97^{*}$ & $543 \pm 153^{*}$ \\
\hline & $\mathrm{LH}+\mathrm{ET}-1$ & $378 \pm 86^{* *}$ & $543 \pm 124$ & $254 \pm 136$ \\
\hline & $\mathrm{LH}+\mathrm{E}_{2}+\mathrm{P}_{4}$ & $452 \pm 79^{* * *}$ & $875 \pm 216^{*}$ & $724 \pm 129^{* *}$ \\
\hline & $\mathrm{LH}+\mathrm{ET}-1+\mathrm{P}_{4}$ & $652 \pm 191^{*}$ & $912 \pm 237^{*}$ & $789 \pm 178^{*}$ \\
\hline & $\mathrm{LH}+\mathrm{ET}-1+\mathrm{E}_{2}+\mathrm{P}_{4}$ & $824 \pm 296^{*}$ & $1095 \pm 317^{* *}$ & $472 \pm 382$ \\
\hline & $\mathrm{OT}+\mathrm{LH}$ & $148 \pm 22$ & $204 \pm 97$ & $232 \pm 123$ \\
\hline & $\mathrm{OT}+\mathrm{LH}+\mathrm{E}_{2}$ & $125 \pm 68$ & $156 \pm 67$ & $148 \pm 89$ \\
\hline & $\mathrm{OT}+\mathrm{E}_{2}+\mathrm{P}_{4}$ & $214 \pm 49$ & $198 \pm 78$ & $157 \pm 69$ \\
\hline & $\mathrm{OT}+\mathrm{E}_{2}+\mathrm{ET}-1$ & $227 \pm 71$ & $215 \pm 40$ & $196 \pm 94$ \\
\hline & $\mathrm{OT}+\mathrm{LH}+\mathrm{ET}-1$ & $98 \pm 67$ & $214 \pm 81$ & $165 \pm 54$ \\
\hline & $\mathrm{OT}+\mathrm{LH}+\mathrm{ET}-1+\mathrm{E}_{2}$ & $169 \pm 72$ & $137 \pm 48$ & $214 \pm 57$ \\
\hline \multirow[t]{13}{*}{$\mathrm{PGF}_{2 \alpha}$} & Control & $118 \pm 24$ & $143 \pm 38$ & $127 \pm 37$ \\
\hline & $\mathrm{P}_{4}+\mathrm{E}_{2}$ & $534 \pm 127$ & $297 \pm 117$ & $199 \pm 84$ \\
\hline & $\mathrm{LH}+\mathrm{E}_{2}$ & $641 \pm 158$ & $547 \pm 182^{*}$ & $817 \pm 224^{\star *}$ \\
\hline & $\mathrm{LH}+\mathrm{ET}-1$ & $741 \pm 217^{*}$ & $689 \pm 117^{*}$ & $717 \pm 173^{* *}$ \\
\hline & $\mathrm{LH}+\mathrm{E}_{2}+\mathrm{P}_{4}$ & $856 \pm 210^{* *}$ & $759 \pm 284^{* *}$ & $987 \pm 214^{* * *}$ \\
\hline & $\mathrm{LH}+\mathrm{ET}-1+\mathrm{P}_{4}$ & $824 \pm 233^{*}$ & $1247 \pm 311^{*}$ & $997 \pm 484$ \\
\hline & $\mathrm{LH}+\mathrm{ET}-1+\mathrm{E}_{2}+\mathrm{P}_{4}$ & $1141 \pm 228^{* * *}$ & $994 \pm 324^{*}$ & $672 \pm 157^{* *}$ \\
\hline & $\mathrm{OT}+\mathrm{LH}$ & $240 \pm 57$ & $314 \pm 137$ & $97 \pm 74$ \\
\hline & $\mathrm{OT}+\mathrm{LH}+\mathrm{E}_{2}$ & $197 \pm 67$ & $257 \pm 114$ & $11 \pm 967$ \\
\hline & $\mathrm{OT}+\mathrm{E}_{2}+\mathrm{P}_{4}$ & $267 \pm 132$ & $158 \pm 86$ & $199 \pm 69$ \\
\hline & $\mathrm{OT}+\mathrm{E}_{2}+\mathrm{ET}-1$ & $183 \pm 81$ & $234 \pm 101$ & $314 \pm 145$ \\
\hline & $\mathrm{OT}+\mathrm{LH}+\mathrm{ET}-1$ & $218 \pm 69$ & $287 \pm 137$ & $217 \pm 98$ \\
\hline & $\mathrm{OT}+\mathrm{LH}+\mathrm{ET}-1+\mathrm{E}_{2}$ & $193 \pm 64$ & $307 \pm 137$ & $267 \pm 161$ \\
\hline \multirow[t]{7}{*}{ ET-1 } & Control & $97 \pm 26$ & $154 \pm 67$ & $115 \pm 29$ \\
\hline & $\mathrm{P}_{4}+\mathrm{E}_{2}$ & $351 \pm 123$ & $341 \pm 184$ & $210 \pm 100$ \\
\hline & $\mathrm{LH}+\mathrm{E}_{2}$ & $489 \pm 98^{* *}$ & $628 \pm 124^{*}$ & $348 \pm 63^{* *}$ \\
\hline & $\mathrm{LH}+\mathrm{E}_{2}+\mathrm{P}_{4}$ & $697 \pm 139$ ** & $845 \pm 214^{*}$ & $821 \pm 121^{* *}$ \\
\hline & $\mathrm{OT}+\mathrm{LH}$ & $167 \pm 68$ & $245 \pm 117$ & $197 \pm 98$ \\
\hline & $\mathrm{OT}+\mathrm{LH}+\mathrm{E}_{2}$ & $219 \pm 91$ & $197 \pm 86$ & $248 \pm 196$ \\
\hline & $\mathrm{OT}+\mathrm{E}_{2}+\mathrm{P}_{4}$ & $212 \pm 87$ & $162 \pm 67$ & $154 \pm 48$ \\
\hline
\end{tabular}

†Indicates the period of treatments.

${ }^{*} P<0 \cdot 05,{ }^{* *} P<0 \cdot 01,{ }^{* * *} P<0 \cdot 001$, significantly different from controls.

The solution was bubbled with a mixture of $95 \% \mathrm{O}_{2}$ and $5 \% \mathrm{CO}_{2}$ and exchanged at a rate of $60 \mathrm{ml} / \mathrm{h}$ using a peristaltic pump. The signals were amplified by an isometric amplifier (RAT-1200 M; Nihon Koden), digitized with an analog-to-digital converter (MacLab/4S; AD Instruments Pty Ltd, Castle Hill, Australia) and stored on a hard disk of a personal computer (Macintosh Power book 190cs; Apple Computer Inc., Cupertino, CA, USA).

The preparation was equilibrated in a bathing solution for $2 \mathrm{~h}$ and then data were recorded for $7 \mathrm{~h}$. After the start of the 7-h experimental period, different hormones were added to the Locke Ringer's solution. The hormones were LH $(100 \mathrm{ng} / \mathrm{ml})$, ET-1 $(10 \mathrm{nmol} / \mathrm{l})$, OT $(10 \mathrm{nmol} / \mathrm{l})$, $\mathrm{PGE}_{2}(100 \mathrm{nmol} / \mathrm{l}), \mathrm{PGF}_{2 \alpha}(10 \mathrm{nmol} / \mathrm{l}), \mathrm{P}_{4}(32 \mathrm{nmol} / \mathrm{l})$ and $E_{2}(37 \mathrm{nmol} / \mathrm{l})$. Because the actions of different hormones on the oviduct contraction were not influenced by side of ovulation or region of the oviduct, the oviduct segments obtained from one cow were randomly allocated for different combinations of treatments $\left(\mathrm{LH}+\mathrm{ET}-1, \mathrm{LH}+\mathrm{OT}, \mathrm{LH}+\mathrm{ET}-1+\mathrm{E}_{2}+\mathrm{P}_{4}\right.$, or $\mathrm{LH}+\mathrm{ET}-$ $\left.1+\mathrm{E}_{2}+\mathrm{P}_{4}+\mathrm{OT}\right)$.

\section{RNA extraction}

Total RNA was extracted from the oviduct $(2 \mathrm{~cm}$ of the whole isthmus) following the protocol of Chomczynski \& Sacchi (1987) using Trizol reagent (Gibco BRL, Gaithersburg, MD, USA). RNA concentration of each 
Table 4 Effects of the infusion of $\mathrm{P}_{4}, \mathrm{E}_{2}, \mathrm{LH}, \mathrm{ET}-1, \mathrm{OT}, \mathrm{PGE}_{2}$ and $\mathrm{PGF}_{2 \alpha}$ on the release of PGs and ET-1 in microdialyzed cow oviducts from the postovulatory phase of the estrous cycle $(n=8-10$, means \pm S.E.M.; control=Ringer's solution only). The data are expressed as percentages of basal secretion $(100 \% ; 0-4 \mathrm{~h})$

\begin{tabular}{|c|c|c|c|c|}
\hline \multirow{2}{*}{ Secretion } & \multirow[b]{2}{*}{ Treatment } & \multicolumn{3}{|c|}{ Incubation period (h) } \\
\hline & & $4-8+$ & $8-12$ & $12-16$ \\
\hline $\begin{array}{l}\text { Secretion } \\
\mathrm{PGE}_{2}\end{array}$ & $\begin{array}{l}\text { Control } \\
\mathrm{P}_{4} \\
\mathrm{E}_{2} \\
\text { LH } \\
\text { ET-1 } \\
\text { OT }\end{array}$ & $\begin{array}{l}101 \pm 33 \\
278 \pm 71 \\
387 \pm 61 \\
313 \pm 81 \\
300 \pm 51 \\
334 \pm 96\end{array}$ & $\begin{array}{l}161 \pm 47 \\
190 \pm 58 \\
292 \pm 91 \\
864 \pm 532^{*} \\
418 \pm 111 \\
268 \pm 134\end{array}$ & $\begin{array}{l}116 \pm 15 \\
157 \pm 53 \\
269 \pm 126 \\
660 \pm 380^{\star} \\
196 \pm 85 \\
112 \pm 39\end{array}$ \\
\hline $\mathrm{PGF}_{2 \alpha}$ & $\begin{array}{l}\text { Control } \\
\mathrm{P}_{4} \\
\mathrm{E}_{2} \\
\text { LH } \\
\text { ET-1 } \\
\text { OT }\end{array}$ & $\begin{aligned} 78 & \pm 14 \\
577 & \pm 138 \\
1015 & \pm 365^{* *} \\
821 & \pm 240^{*} \\
508 & \pm 79^{*} \\
238 & \pm 23\end{aligned}$ & $\begin{aligned} 80 & \pm 13 \\
867 & \pm 295 \\
820 & \pm 417 \\
1337 & \pm 460 \\
1287 & \pm 318^{\star *} \\
243 & \pm 126\end{aligned}$ & $\begin{aligned} 71 & \pm 22 \\
516 & \pm 149 \\
599 & \pm 338 \\
933 & \pm 339^{*} \\
671 & \pm 141^{*} \\
180 & \pm 73\end{aligned}$ \\
\hline ET-1 & $\begin{array}{l}\text { Control } \\
\mathrm{P}_{4} \\
\mathrm{E}_{2} \\
\mathrm{LH} \\
\mathrm{OT} \\
\mathrm{PGE}_{2} \\
\text { PGF }_{2 \alpha}\end{array}$ & $\begin{aligned} 85 & \pm 13 \\
224 & \pm 81 \\
291 & \pm 42 \\
543 & \pm 123^{* * *} \\
291 & \pm 33 \\
109 & \pm 22 \\
93 & \pm 14\end{aligned}$ & $\begin{aligned} 91 & \pm 11 \\
233 & \pm 82 \\
446 & \pm 165^{*} \\
609 & \pm 119^{* *} \\
209 & \pm 39 \\
158 & \pm 94 \\
89 & \pm 28\end{aligned}$ & $\begin{aligned} 94 & \pm 14 \\
193 & \pm 44 \\
93 & \pm 36 \\
748 & \pm 223^{* * *} \\
146 & \pm 32 \\
107 & \pm 18 \\
154 & \pm 79\end{aligned}$ \\
\hline
\end{tabular}

†Indicates the period of treatments.

${ }^{*} P<0 \cdot 05,{ }^{*} P<0 \cdot 01,{ }^{* *} P<0 \cdot 001$, significantly different from controls.

sample was determined by UV-spectroscopy (OD $260 \mathrm{~nm})$. Integrity of total RNA was controlled by agarose/formaldehyde gel electrophoresis followed by ethidium bromide staining.

\section{Reverse transcription-polymerase chain reaction (RT-PCR)}

Four micrograms total RNA were subjected to firststrand cDNA synthesis using $2.5 \mu \mathrm{M}$ random hexamers (Pharmacia, Freiburg, Germany) and the Superscript II reverse transcriptase (Gibco BRL) in a final volume of $60 \mu \mathrm{l}$ as previously described (Gabler et al. 1998). The following commercially synthesized primers (Gibco BRL) were used to amplify specific bovine transcripts: ET-1 (333 bp) forward: 5' TCC CCA GAA TGG ATT ATT TCC 3', reverse: $5^{\prime}$ TCT TCC TGT GGA CTG TCG C 3'; ET-R type A (329 bp) forward: 5' TGC AGA AGT CCT CAG TGG G 3', reverse: 5' GAT CGC AGT GCA CAC CAG 3'; ET-R type B (296 bp) forward: 5' AAA CTG AGA ATC TGC TTG CTC C 3', reverse: 5' AGA GTG AGC TTC AAA ATC CTG C $3^{\prime}$. The predicted size of each RT-PCR product is shown in parentheses.

Aliquots of cDNA ( $5 \mu \mathrm{l}$ ) were amplified with $0.5 \mathrm{U}$ Taq polymerase (QIAGEN, Valencia, CA, USA) in a thermal Cycler (Biometra, Goettingen, Germany) in a final volume of $25 \mu \mathrm{l}$, as previously described (Gabler et al. 1997). The following amplification programs were applied for
ET-1 (40 cycles at $94{ }^{\circ} \mathrm{C}$ and $60{ }^{\circ} \mathrm{C}, 1$ min each), as well as 29 cycles for ET-R type A and 31 cycles for ET-R type B $\left(94{ }^{\circ} \mathrm{C}\right.$ and $60{ }^{\circ} \mathrm{C}, 1 \mathrm{~min}$ each) respectively. An $18 \mathrm{~S}$ rRNA-PCR (324 bp) was performed to control the integrity of the RNA, as well as the efficiency of the reverse transcription for each sample. The $18 \mathrm{~S}$ primers and the appropriate competitors were used in a ratio of 2:3 (Ambion, Austin, TX, USA). Twenty-four amplification cycles were performed $\left(94^{\circ} \mathrm{C}, 60{ }^{\circ} \mathrm{C}\right.$ and $72{ }^{\circ} \mathrm{C}, 30 \mathrm{~s}$ each). As a negative control, water was used instead of RNA for the RT-PCR to exclude any contamination from buffers and tubes. Furthermore, an additional control was performed omitting the reverse transcriptase to exclude any amplification of genomic DNA.

Aliquots of $5 \mu \mathrm{l}$ per reaction were run on $1.5 \%(\mathrm{w} / \mathrm{v})$ agarose gels containing $1.0 \mu \mathrm{g} / \mathrm{ml}$ ethidium bromide. To confirm the identity of the PCR products, each PCR product was sequenced. The resultant band intensities were scanned by a video documentation system (Eagle Eye, Stratagene, La Jolla, CA, USA) and analyzed with the Gel-Pro Analyzer software (Media Cybernetics, Silver Spring, MD, USA).

\section{Statistical analysis}

The mean concentrations of each of the substances in the first $4 \mathrm{~h}$ of incubation (perfusion with Ringer's solution 
Table 5 Effects of combined treatment of hormonal stimulation on the release of PGs and ET-1 in microdialyzed cow oviducts collected from the postovulatory phase of the estrous cycle ( $n=4-5$, means \pm S.E.M.; control=Ringer's solution only). The data are expressed as percentages of basal secretion $(100 \% ; 0-4 \mathrm{~h})$

\begin{tabular}{|c|c|c|c|c|}
\hline & \multirow[b]{2}{*}{ Treatment } & \multicolumn{3}{|c|}{ Incubation period $(\mathrm{h})$} \\
\hline & & $\overline{4-8 \dagger}$ & $8-12$ & $12-16$ \\
\hline & & & & \\
\hline \multirow[t]{13}{*}{$\mathrm{PGE}_{2}$} & Control & $98 \pm 20$ & $89 \pm 21$ & $100 \pm 21$ \\
\hline & $\mathrm{P}_{4}+\mathrm{E}_{2}$ & $324 \pm 82$ & $464 \pm 88$ & $285 \pm 58$ \\
\hline & $\mathrm{LH}+\mathrm{E}_{2}$ & $1243 \pm 495^{* \star *}$ & $1006 \pm 397^{*}$ & $968 \pm 533^{*}$ \\
\hline & $\mathrm{LH}+\mathrm{ET}-1$ & $269 \pm 57$ & $909 \pm 475^{* *}$ & $930 \pm 201^{* * *}$ \\
\hline & $\mathrm{LH}+\mathrm{E}_{2}+\mathrm{P}_{4}$ & $267 \pm 28$ & $515 \pm 119 * *$ & $368 \pm 122$ \\
\hline & $\mathrm{LH}+\mathrm{ET}-1+\mathrm{P}_{4}$ & $265 \pm 185$ & $873 \pm 249 * *$ & $584 \pm 158^{*}$ \\
\hline & $\mathrm{LH}+\mathrm{ET}-1+\mathrm{E}_{2}+\mathrm{P}_{4}$ & $401 \pm 69^{*}$ & $907 \pm 103^{* *}$ & $890 \pm 282^{* * *}$ \\
\hline & $\mathrm{OT}+\mathrm{LH}$ & $207 \pm 37$ & $164 \pm 61$ & $122 \pm 41$ \\
\hline & $\mathrm{OT}+\mathrm{LH}+\mathrm{E}_{2}$ & $276 \pm 54$ & $363 \pm 133$ & $250 \pm 49$ \\
\hline & $\mathrm{OT}+\mathrm{E}_{2}+\mathrm{P}_{4}$ & $125 \pm 28$ & $249 \pm 53$ & $164 \pm 47$ \\
\hline & $\mathrm{OT}+\mathrm{E}_{2}+\mathrm{ET}-1$ & $176 \pm 44$ & $148 \pm 22$ & $96 \pm 20$ \\
\hline & $\mathrm{OT}+\mathrm{LH}+\mathrm{ET}-1$ & $142 \pm 66$ & $146 \pm 38$ & $86 \pm 20$ \\
\hline & $\mathrm{OT}+\mathrm{LH}+\mathrm{ET}-1+\mathrm{E}_{2}$ & $211 \pm 44$ & $234 \pm 21$ & $122 \pm 20$ \\
\hline \multirow[t]{13}{*}{$\mathrm{PGF}_{2 \alpha}$} & Control & $122 \pm 12$ & $114 \pm 15$ & $117 \pm 36$ \\
\hline & $\mathrm{P}_{4}+\mathrm{E}_{2}$ & $910 \pm 270^{* *}$ & $838 \pm 348^{*}$ & $898 \pm 363^{*}$ \\
\hline & $\mathrm{LH}+\mathrm{E}_{2}$ & $1438 \pm 358$ & $949 \pm 282^{*}$ & $613 \pm 204^{*}$ \\
\hline & $\mathrm{LH}+\mathrm{ET}-1$ & $956 \pm 320^{*}$ & $1431 \pm 517^{*}$ & $2075 \pm 970^{*}$ \\
\hline & $\mathrm{LH}+\mathrm{E}_{2}+\mathrm{P}_{4}$ & $1351 \pm 402^{* *}$ & $1197 \pm 433^{*}$ & $2003 \pm 693 * *$ \\
\hline & $\mathrm{LH}+\mathrm{ET}-1+\mathrm{P}_{4}$ & $801 \pm 233^{*}$ & $2002 \pm 458^{* * *}$ & $609 \pm 201$ \\
\hline & $\mathrm{LH}+\mathrm{ET}-1+\mathrm{E}_{2}+\mathrm{P}_{4}$ & $1225 \pm 133^{* *}$ & $2269 \pm 424^{* * *}$ & $1233 \pm 742$ \\
\hline & $\mathrm{OT}+\mathrm{LH}$ & $568 \pm 240$ & $771 \pm 199$ & $560 \pm 143$ \\
\hline & $\mathrm{OT}+\mathrm{LH}+\mathrm{E}_{2}$ & $402 \pm 374$ & $469 \pm 359$ & $550 \pm 378$ \\
\hline & $\mathrm{OT}+\mathrm{E}_{2}+\mathrm{P}_{4}$ & $253 \pm 123$ & $322 \pm 184$ & $143 \pm 20$ \\
\hline & $\mathrm{OT}+\mathrm{E}_{2}+\mathrm{ET}-1$ & $249 \pm 82$ & $324 \pm 192$ & $211 \pm 93$ \\
\hline & $\mathrm{OT}+\mathrm{LH}+\mathrm{ET}-1$ & $139 \pm 12$ & $156 \pm 37$ & $159 \pm 62$ \\
\hline & $\mathrm{OT}+\mathrm{LH}+\mathrm{ET}-1+\mathrm{E}_{2}$ & $420 \pm 417$ & $577 \pm 349$ & $266 \pm 183$ \\
\hline \multirow[t]{7}{*}{$\mathrm{ET}-1$} & Control & $102 \pm 16$ & $99 \pm 59$ & $74 \pm 23$ \\
\hline & $\mathrm{P}_{4}+\mathrm{E}_{2}$ & $492 \pm 116^{\star *}$ & $491 \pm 169^{*}$ & $433 \pm 105^{*}$ \\
\hline & $\mathrm{LH}+\mathrm{E}_{2}$ & $553 \pm 121^{* * *}$ & $455 \pm 96^{* *}$ & $248 \pm 47$ \\
\hline & $\mathrm{LH}+\mathrm{E}_{2}+\mathrm{P}_{4}$ & $244 \pm 31^{* \star}$ & $398 \pm 41^{* *}$ & $301 \pm 132$ \\
\hline & $\mathrm{OT}+\mathrm{LH}$ & $66 \pm 27$ & $108 \pm 28$ & $67 \pm 27$ \\
\hline & $\mathrm{OT}+\mathrm{LH}+\mathrm{E}_{2}$ & $123 \pm 23$ & $81 \pm 37$ & $97 \pm 58$ \\
\hline & $\mathrm{OT}+\mathrm{E}_{2}+\mathrm{P}_{4}$ & $80 \pm 25$ & $79 \pm 28$ & $205 \pm 94$ \\
\hline
\end{tabular}

†Indicates the period of treatments.

${ }^{*} P<0 \cdot 05,{ }^{* *} P<0 \cdot 01,{ }^{* * *} P<0 \cdot 001$, significantly different from controls.

only) were used to calculate the individual baseline for each hormone, because of a large variation in the absolute amount of hormones released into each of the microdialysis capillary membranes implanted in the various oviducts. All values were expressed as a percentage of the corresponding baseline. The coefficient of variation in the absolute concentration of each hormone in the microdialysis perfusates collected from different oviducts during the first $4 \mathrm{~h}$ (baseline) was as follows: $\mathrm{PGE}_{2}=84-304 \%, \mathrm{PGF}_{2 \alpha}=77-$ $269 \%$, and $\mathrm{ET}-1=41-73 \%$. This transformation enables an evaluation of relative changes of hormonal values between different oviducts. The effects of the infused substances on the release of $\mathrm{PGE}_{2}, \mathrm{PGF}_{2 \alpha}$, and ET-1 were compared with control values obtained during the same time period using ANOVA followed by Duncan's new multiple range test. Differences were considered significant at a probability less than $5 \%(P<0 \cdot 05)$.

Amplitude and frequency of oviduct contractions in the control and treatment groups were averaged over 10-min intervals and analyzed using the MacLab Chat data acquisition/analysis program (1997; MacLab, AD Instruments). The basal oviduct amplitude varied considerably among individual oviducts. Therefore, the baseline of oviduct amplitude (defined as 100\%) was calculated by averaging the data for the first hour, and the amplitude of each 10-min interval was then expressed as a percentage of this individual baseline. As the basal amplitude was very stable, the effects of the added substances on the 
Table 6 Effects of the infusion of LH, ET-1, OT, LH+OT and LH+ET-1+OT in microdialyzed cow oviducts from the luteal phase of the estrous cycle $(n=4-5$, means \pm S.E.M.; control=Ringer's solution only). The data are expressed as percentages of basal secretion (100\%; 0-4 h)

\begin{tabular}{|c|c|c|c|c|}
\hline \multirow{4}{*}{ Secretion } & \multirow[b]{2}{*}{ Treatment } & \multicolumn{3}{|c|}{ Incubation period (h) } \\
\hline & & \multirow[t]{2}{*}{$4-8 \dagger$} & \multirow[t]{2}{*}{$8-12$} & \multirow[t]{2}{*}{$12-16$} \\
\hline & & & & \\
\hline & Control & $122 \pm 21$ & $192 \pm 52$ & $122 \pm 52$ \\
\hline & $\mathrm{LH}$ & $183 \pm 29$ & $270 \pm 63$ & $271 \pm 23$ \\
\hline & $\mathrm{ET}-1$ & $252 \pm 68$ & $201 \pm 37$ & $214 \pm 85$ \\
\hline & OT & $191 \pm 33$ & $256 \pm 113$ & $133 \pm 33$ \\
\hline & $\mathrm{LH}+\mathrm{ET}-1$ & $165 \pm 15$ & $216 \pm 49$ & $131 \pm 20$ \\
\hline & $\mathrm{LH}+\mathrm{OT}$ & $235 \pm 59$ & $322 \pm 48$ & $190 \pm 29$ \\
\hline & $\mathrm{LH}+\mathrm{ET}-1+\mathrm{OT}$ & $467 \pm 162^{*}$ & $426 \pm 162$ & $509 \pm 164^{*}$ \\
\hline \multirow{7}{*}{$\mathrm{PGF}_{2 \alpha}$} & Control & $102 \pm 32$ & $93 \pm 10$ & $113 \pm 16$ \\
\hline & $\mathrm{LH}$ & $376 \pm 238$ & $186 \pm 69$ & $377 \pm 196$ \\
\hline & $\mathrm{ET}-1$ & $416 \pm 95$ & $203 \pm 60$ & $352 \pm 64$ \\
\hline & OT & $230 \pm 71$ & $391 \pm 98$ & $155 \pm 40$ \\
\hline & $\mathrm{LH}+\mathrm{ET}-1$ & $396 \pm 223$ & $305 \pm 149$ & $309 \pm 113$ \\
\hline & $\mathrm{LH}+\mathrm{OT}$ & $64 \pm 16$ & $157 \pm 44$ & $194 \pm 71$ \\
\hline & $\mathrm{LH}+\mathrm{ET}-1+\mathrm{OT}$ & $169 \pm 90$ & $279 \pm 66$ & $252 \pm 100$ \\
\hline \multirow[t]{4}{*}{ ET-1 } & Control & $57 \pm 28$ & $144 \pm 42$ & $97 \pm 50$ \\
\hline & $\mathrm{LH}$ & $153 \pm 25^{*}$ & $221 \pm 65$ & $223 \pm 62$ \\
\hline & OT & $130 \pm 48$ & $267 \pm 100$ & $208 \pm 95$ \\
\hline & $\mathrm{LH}+\mathrm{OT}$ & $110 \pm 7$ & $193 \pm 73$ & $145 \pm 57$ \\
\hline
\end{tabular}

†Indicates the period of treatments

${ }^{*} P<0 \cdot 05$, significantly different from controls.

contraction were compared with control values obtained during the same time point using ANOVA followed by Duncan's new multiple range test.

Statistical analyses of ET-1, ET-R A and ET-R B mRNA were corrected with the signal intensities of the 18S rRNA. The expression of ET-1, ET-R A and ET-R $\mathrm{B}$ during different phases of the estrous cycle was analyzed using ANOVA. When ANOVA showed significant differences, the Tukey-Kramer test was used to test significance. Probabilities less than $0.05(P<0.05)$ were considered significant.

\section{Results}

The basal release of substances in the microdialysis system, as well as the spontaneous amplitude and frequency of the oviduct contraction in the control group were constant and stable during the experimental periods. The basal release of all substances measured and the spontaneous amplitude and frequency of the oviduct contractions were higher $(P<0 \cdot 05)$ during the follicular and postovulatory phases than during the luteal phase (Table 1). Moreover, the contraction pattern of the oviducts was more regular during the follicular and postovulatory phases than during the luteal phase.
Effect of substance infusion on PG and ET-1 secretion from microdialyzed oviduct from the follicular and postovulatory phases

$\mathrm{E}_{2}$ stimulated $\mathrm{PGF}_{2 \alpha}$ release $(4-16 \mathrm{~h} ; P<0 \cdot 05$ to $P<0 \cdot 01)$ and ET-1 release $(8-12 \mathrm{~h} ; P<0 \cdot 01)$ in follicular phase oviducts, while $\mathrm{P}_{4}$ only stimulated $\mathrm{PGF}_{2 \alpha}$ secretion (812 h; $P<0 \cdot 05$; Table 2). ET-1 stimulated $\mathrm{PGF}_{2 \alpha}$ release $(P<0 \cdot 01)$ during infusion, although PG showed no effect on the ET-1 secretion. LH stimulated PG and ET-1 secretion $(P<0.05$ to $P<0.01)$, and OT had no effect on PG or ET-1 release (Table 2). The infusion of LH with different combinations of $\mathrm{P}_{4}, \mathrm{E}_{2}$ and/or ET-1 resulted in an acute and continuous increase in the release of $P G$ and ET-1 $(P<0.05$ to $P<0 \cdot 001)$. However, simultaneous administration of $\mathrm{OT}$ with different combinations of $\mathrm{LH}$, $\mathrm{P}_{4}, \mathrm{E}_{2}$ and ET-1 did not alter the basal PG or ET-1 release (Table 3).

A 4-h infusion of $\mathrm{E}_{2}$ induced an acute $\mathrm{PGF}_{2 \alpha}$ release $(P<0 \cdot 01)$, and $\mathrm{E}_{2}$ stimulated ET-1 release $8-12 \mathrm{~h}$ $(P<0 \cdot 05)$ in postovulatory phase oviducts (Table 4$)$. The ET-1 infusion stimulated $\mathrm{PGF}_{2 \alpha}$ release in the oviducts from the postovulatory phase $(P<0 \cdot 05$ to $P<0 \cdot 01)$. LH stimulated $\mathrm{PG}$ secretion $(P<0 \cdot 05)$ and ET-1 secretion $(P<0 \cdot 01$ to $P<0 \cdot 001)$, which was sustained throughout the experimental period (Table 4$)$. The combined infusion of $\mathrm{P}_{4}$ and $\mathrm{E}_{2}$ significantly $(P<0 \cdot 05$ to $P<0 \cdot 01)$ increased 


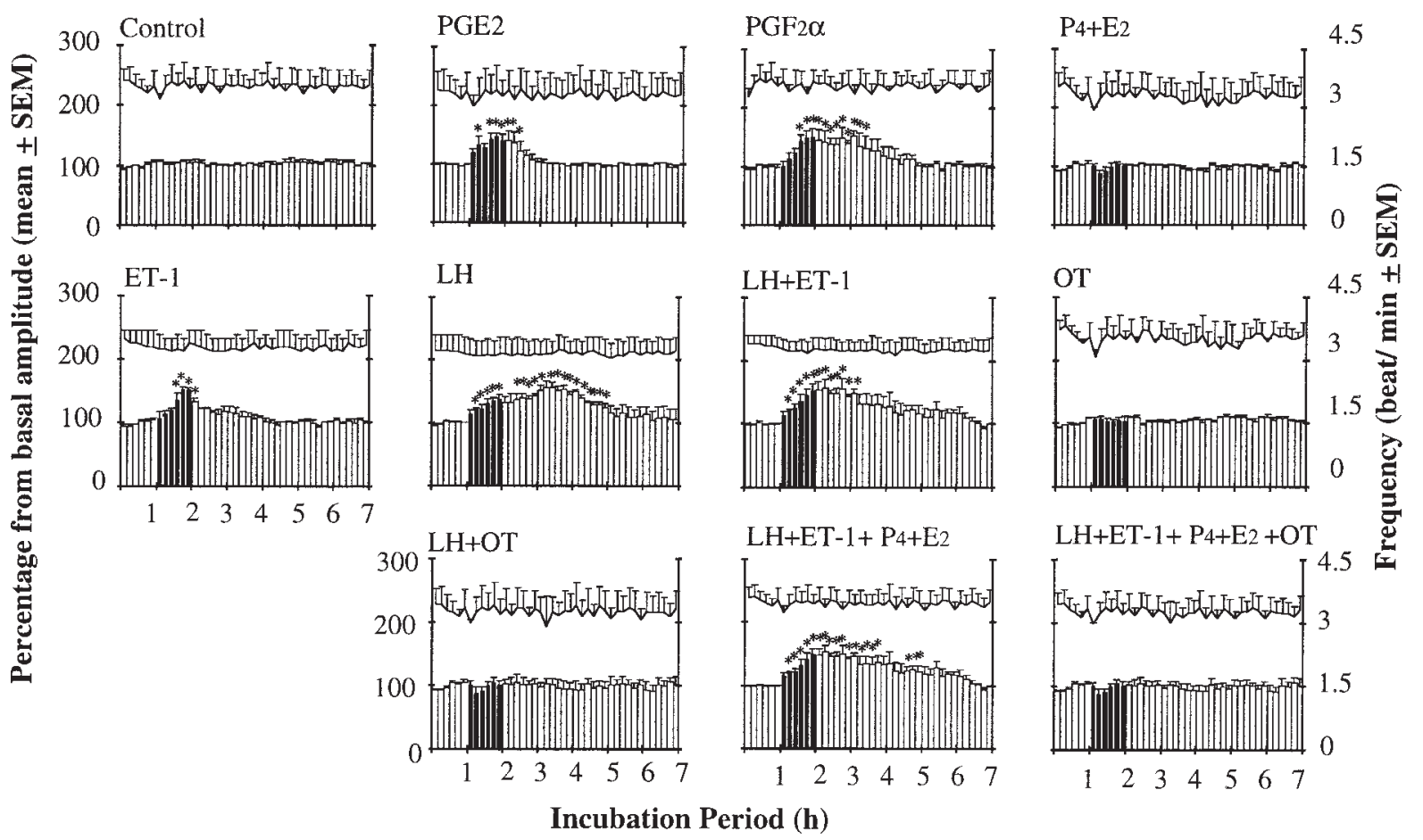

Figure 2 Effect of different substances on the amplitude (bars) and frequency (line) of the contractions of the oviducts collected during the follicular phase $\left(n=4-5\right.$, means \pm S.E.M.). Solid bars indicate the period of treatment. ${ }^{*} P<0 \cdot 05$, significantly different from controls at the same time point.

$\mathrm{PGF}_{2 \alpha}$ and ET-1 production $(P<0 \cdot 05$ to $0 \cdot 01$; Table 5). Combined infusion of LH with $\mathrm{P}_{4}, \mathrm{E}_{2}$ and/or ET-1 resulted in an increase in PG and ET- 1 release $(P<0 \cdot 05$ to $P<0 \cdot 001)$. However, simultaneous administration of OT with different combinations of $\mathrm{LH}, \mathrm{P}_{4}, \mathrm{E}_{2}$ and ET-1 abolished stimulation of PG and ET-1 release (Table 5).

\section{Effect of substance infusion on PG and ET-1 secretion from microdialyzed oviduct from the luteal phase}

The basal release of PG and ET-1 during the luteal phase was significantly lower $(P<0 \cdot 05)$ than that of the follicular and postovulatory phases (Table 1$)$. Although some substances stimulated the production of $\mathrm{PGE}_{2}, \mathrm{PGF}_{2 \alpha}$ and ET-1, the magnitude of this stimulation was significantly lower $(P<0 \cdot 05)$ than that observed during the follicular or postovulatory phases (Table 6).

\section{Effect of substances on the oviduct contraction in vitro}

Administration of LH, ET-1, $\mathrm{PGE}_{2}, \mathrm{PGF}_{2 \alpha}, \mathrm{LH}+\mathrm{ET}-1$ and $\mathrm{LH}+\mathrm{ET}-1+\mathrm{P}_{4}+\mathrm{E}_{2}$ significantly increased $(P<0 \cdot 05)$ the amplitude of oviduct contraction in both follicular and postovulatory phase oviducts. The stimulation of the amplitude of oviduct contraction by the combination of $\mathrm{LH}+\mathrm{ET}-1+\mathrm{P}_{4}+\mathrm{E}_{2}$ during the postovulatory and follicular phases, as well as the stimulation by $\mathrm{LH}$ during the follicular phase persisted up to $4 \mathrm{~h}$ after the withdrawal of treatment. However, stimulation by other substances or combinations lasted for $1-2 \mathrm{~h}$ after the treatments. The combination of OT with other substances completely blocked contractile amplitude. None of these treatments altered the basal frequency of the oviduct contraction during any phase of the estrous cycle (Figs 2 and 3). Moreover, in luteal phase oviducts, the above treatments did not affect the amplitude or frequency of contractions (Fig. 4).

\section{Expression of $m R N A$ for ET-1 and ET-R types $A$ and $B$}

Specific transcripts for ET-1, ET-R A and ET-R B were detected in the bovine oviduct. Each PCR product showed 100\% homology to the known bovine genes after sequencing. Quantity and quality of each RNA sample were verified after denaturing agarose gel electrophoresis, as well as comparative RT-PCR for the ribosomal 18S rRNA (Fig. 5). The relative signal intensities for PCR products specific for ET-1, ET-R A and ET-R B were assessed after correction based on the 18S rRNA signal intensities. The same expression pattern for both endothelin receptors, as well as for the ligand endothelin was found during the estrous cycle: highest expression during the phase after ovulation, lower expression during the luteal phase and the lowest expression before ovulation (Fig. 6). 

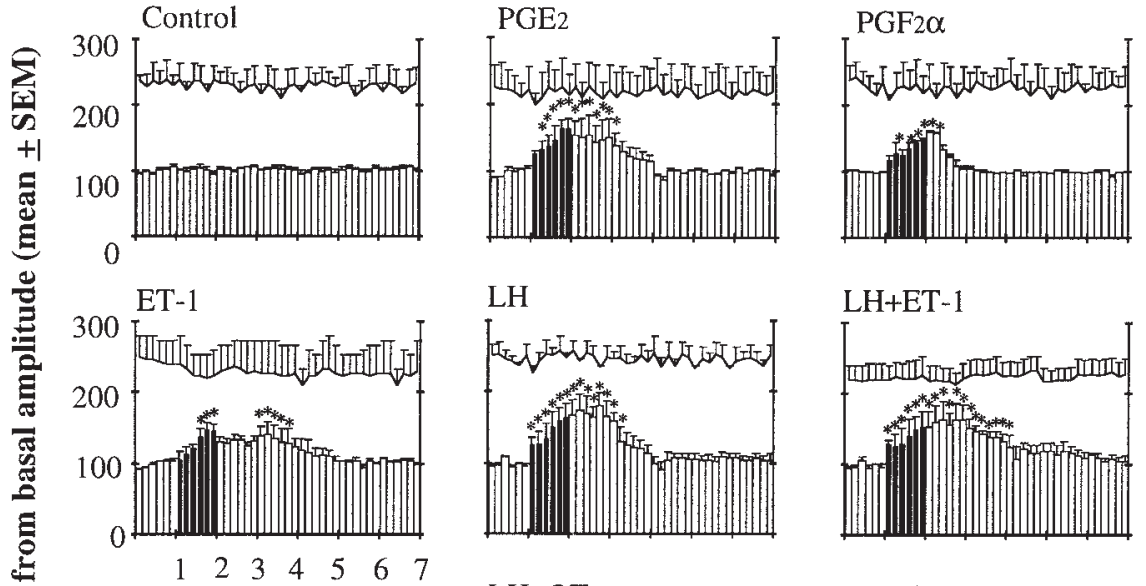

LH
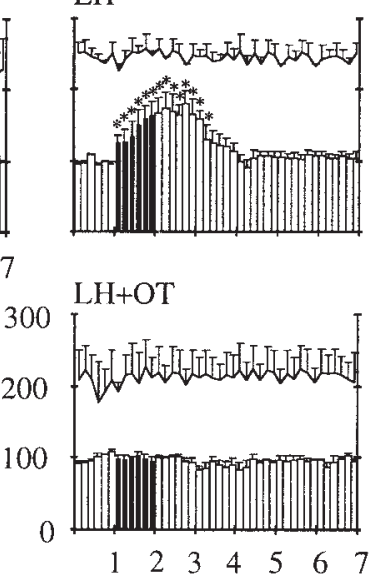
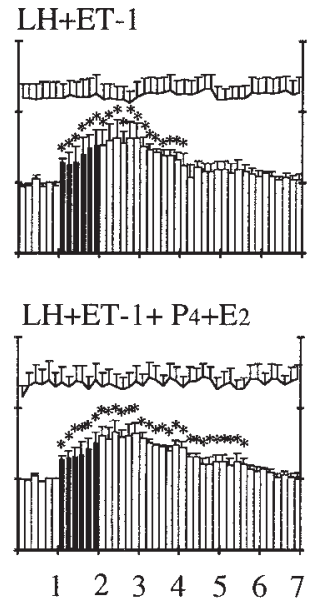
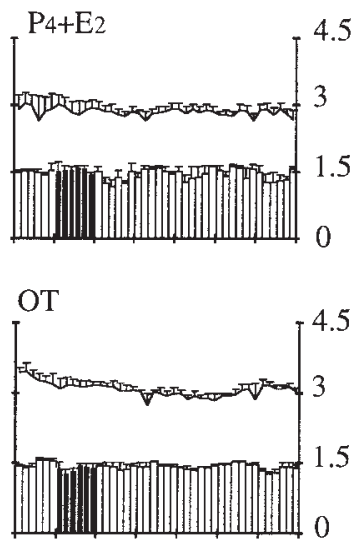

$\mathrm{LH}+\mathrm{ET}-1+\mathrm{P} 4+\mathrm{E} 2+\mathrm{OT}$

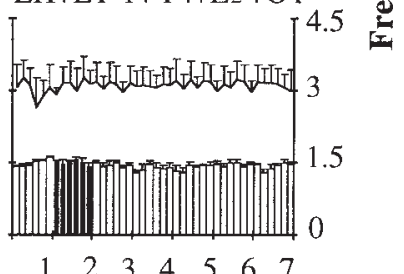

Incubation Period (h)

Figure 3 Effect of different substances on the amplitude (bars) and frequency (line) of the contractions of the oviducts collected during the postovulatory phase. $\left(n=4-5\right.$, means \pm S.E.M.) Solid bars indicate the period of treatment. ${ }^{*} P<0 \cdot 05$, significantly different from controls at the same time point.

This expression pattern was significant for ET and ET-R $\mathrm{B}$ as shown by ANOVA. The difference of the ET-R A expression during the postovulatory and follicular phases approached significance $(P=0 \cdot 0642)$.

\section{Discussion}

The results in this study indicate that $\mathrm{LH}$ enhances the in vitro secretion of $\mathrm{PGE}_{2}, \mathrm{PGF}_{2 \alpha}$ and ET-1, as well as the contractile amplitude of bovine oviducts from the follicular and postovulatory periods, but not those from the luteal period. Furthermore, ET-1 stimulated the release of $\mathrm{PGE}_{2}$ and $\mathrm{PGF}_{2 \alpha}$ from the oviduct epithelial cells in the microdialysis experiment. Our recent observations of the cyclic changes in local content of PG and ET-1 in the bovine oviduct revealed that the oviduct contains the highest concentrations of PG and ET-1 during the periovulatory period (Wijayagunawardane et al. 1998). This was confirmed by the present results in which the secreted levels of PG and ET-1 were higher during the follicular and postovulatory periods than during the luteal phase in the microdialysis study. Furthermore, we found that ET-R A and $\mathrm{B}$ mRNAs were highly expressed after ovulation, suggesting that the functional ET system is primarily active after ovulation in vivo.
The demonstration of LH/human chorionic gonadotropin (hCG) receptors in the oviduct of woman (Lei et al. 1993, Han et al. 1996), sow (Derecka et al. 1995) and cow (Sun et al. 1997) suggested a direct involvement of LH/hCG in early pregnancy events (Lei et al. 1993, Derecka et al. 1995, Han et al. 1996). It was reported that LH increased 5-lipoxygenase, cyclooxygenase (COX)-1 and COX-2 enzymes in the human oviduct (Lei et al. 1993, Han et al. 1996), and increased PG release in human umbilical cord (Rao et al. 1993), cultured endothelial cells (Shemesh et al. 1997), and human fetal membranes (Toth et al. 1996). Moreover, LH activates protein kinase (PK) A and PKC in porcine myometrial smooth muscle cells (Kisielewska et al. 1996) and such an activation of PK results in increased ET-1 mRNA levels in cultured bovine endothelial cells (Emori et al. 1991). The results of the present study clearly indicated that $\mathrm{LH}$ stimulates the secretion of $\mathrm{PGE}_{2}, \mathrm{PGF}_{2 \alpha}$ and ET-1 in the oviduct, and that $\mathrm{LH}$ also increases oviduct contraction. We postulate that the LH-induced oviduct contraction, at least in part, is due to the increased secretion of $\mathrm{PGE}_{2}, \mathrm{PGF}_{2 \alpha}$ and ET-1.

PGs are reported to modulate oviduct contraction, through effects of E-series PG on relaxation of smooth muscle and of F-series PG on contraction (Spilman \& Harper 1975). Human oviduct musculature responds to 

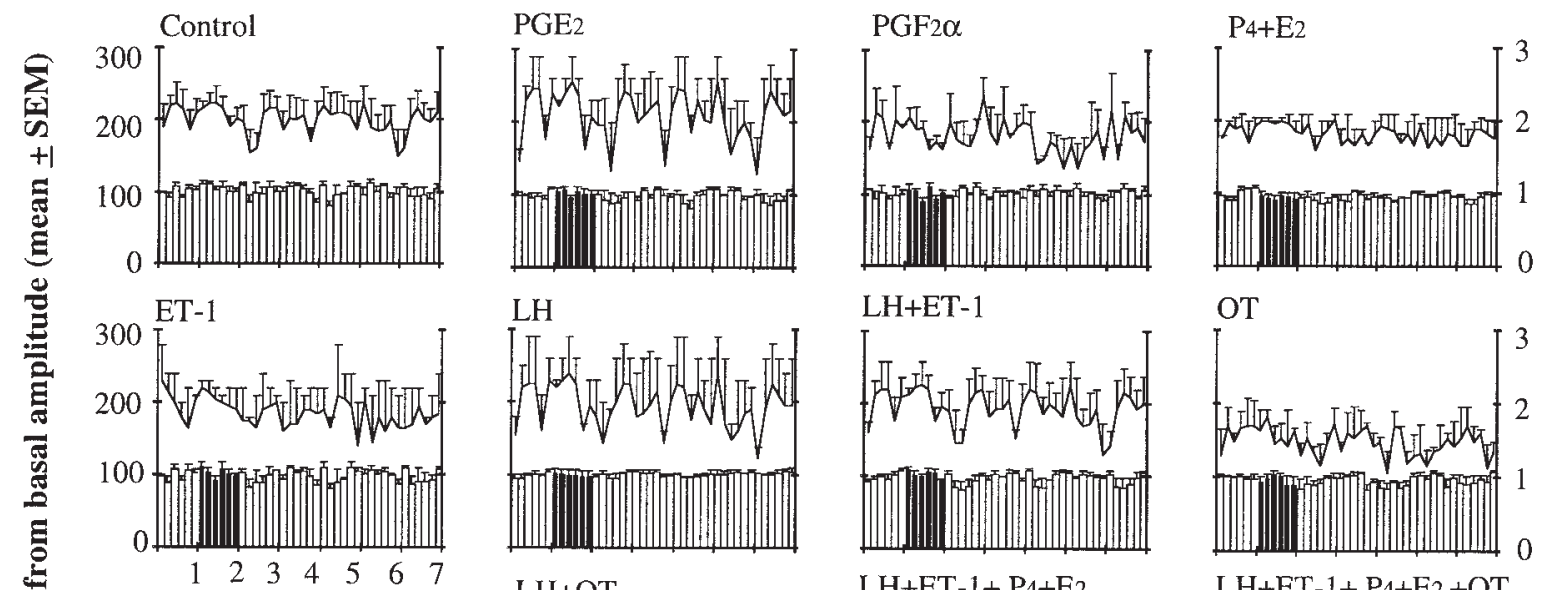

LH
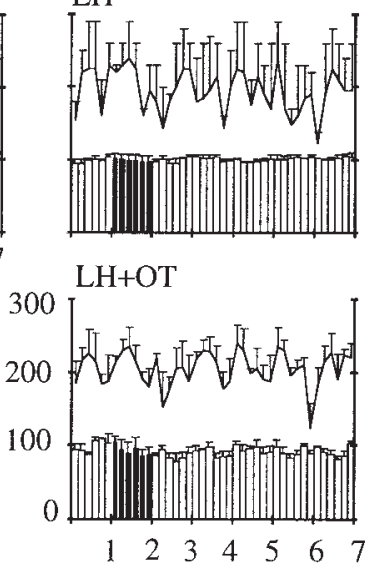

LH+ET-1

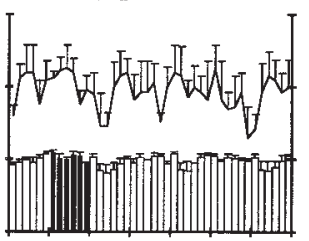

$\mathrm{LH}+\mathrm{ET}-1+\mathrm{P} 4+\mathrm{E} 2$

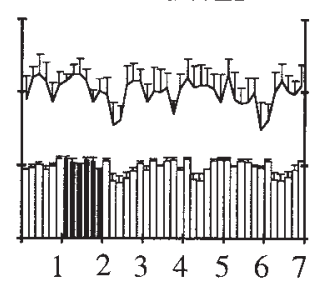

OT

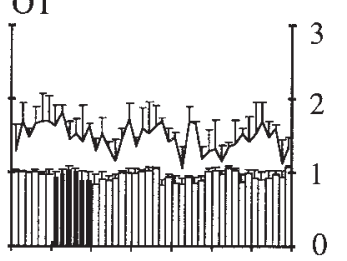

$\mathrm{LH}+\mathrm{ET}-1+\mathrm{P} 4+\mathrm{E} 2+\mathrm{OT}$

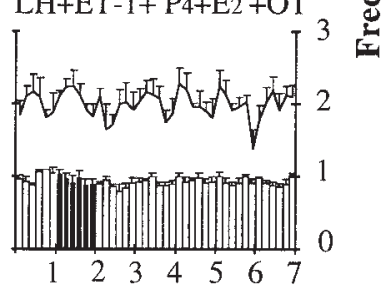

Incubation Period (h)

Figure 4 Effect of different substances on the amplitude (bars) and frequency (line) of the contractions of the oviducts collected during the luteal phase $(n=4-5$, means \pm S.E.M.). Solid bars indicate the period of treatment.

$\mathrm{PGF}_{2 \alpha}$ by increasing tonus (Atanackovic et al. 1983), and $\mathrm{PGF}_{2 \alpha}$ is recognized as a prerequisite for maintenance of normal tubal contractions (Lindblom \& Andersson 1985). The $\mathrm{PGF}_{2 \alpha}$ receptors in the adult rat oviduct predominated during the periovulatory period (Orlicky \& Williams-Skipp 1993) and the response of the oviduct to $\mathrm{PGF}_{2 \alpha}$ increased markedly following ovulation in marmoset monkeys (Barbosa et al. 1980). Also, $\mathrm{PGE}_{2}$ treatment hastened the oviduct transport of equine embryos (Weber et al. 1991). The direct stimulation of the amplitude of oviduct contraction by PG during the follicular and postovulatory phases observed in this study may be due to the increased sensitivity as a result of an increase in the number of binding sites during this period.

ET-1-induced contractile responses and increased oscillation frequency in rat uterine smooth muscle appear to be mediated through ET-R type A (Tsunoda et al. 1993). Rosselli et al. (1994) observed ET-1 production by bovine oviduct epithelial cells and hypothesized the possible involvement of ET-1 in the muscular oviduct wall contraction. Moreover, ET-1 acts in bovine oviduct arteries by directly activating a homogenous population of ET-R type A in smooth muscle (Labadia et al. 1997). In the present study, oviduct expression of mRNA for ET-R, both $\mathrm{A}$ and $\mathrm{B}$ types, showed a similar pattern with the highest mRNA levels observed during the postovulatory phase. Smooth muscle cell is the predominant cell type in the oviduct isthmus and both ET receptors are believed to be primarily expressed in smooth muscle cells. Because the maximum stimulatory effect of ET-1 was observed during the postovulatory phase in the present study, it may be the result of elevated ET-R concentration in the oviduct smooth muscles during this period. It was reported that ovarian steroids play a role in the control of expression of ET-R in human endometrium (Collett et al. 1996) and that endogenous $E_{2}$ increases the affinity of high-affinity ET-R in coronary arterial smooth muscle of female pigs (Barber et al. 1996). Moreover, we recently reported that the local distribution of $E_{2}$ in the cow oviduct is highest during the follicular phase (Wijayagunawardane et al. 1998). Therefore, the stimulatory effect of ET-1 on oviduct secretion and contraction during the follicular phase, even with the lowest ET-R levels in the oviduct, may be due to increases in the affinity of the ET-R by the elevated local levels of $E_{2}$ in the oviduct.

Surprisingly, OT completely blocked increases in secretion of PG and ET-1 and increases in the contractile amplitude that were stimulated by different combinations of substances used in the present study. This is the first evidence that OT may act locally on oviduct function as a suppressor. In sheep OT is locally recirculated to the oviduct in a higher concentration than is supplied by peripheral blood (Schramm et al. 1986) and in women local oviductal tissue levels of OT exceeded its plasma 

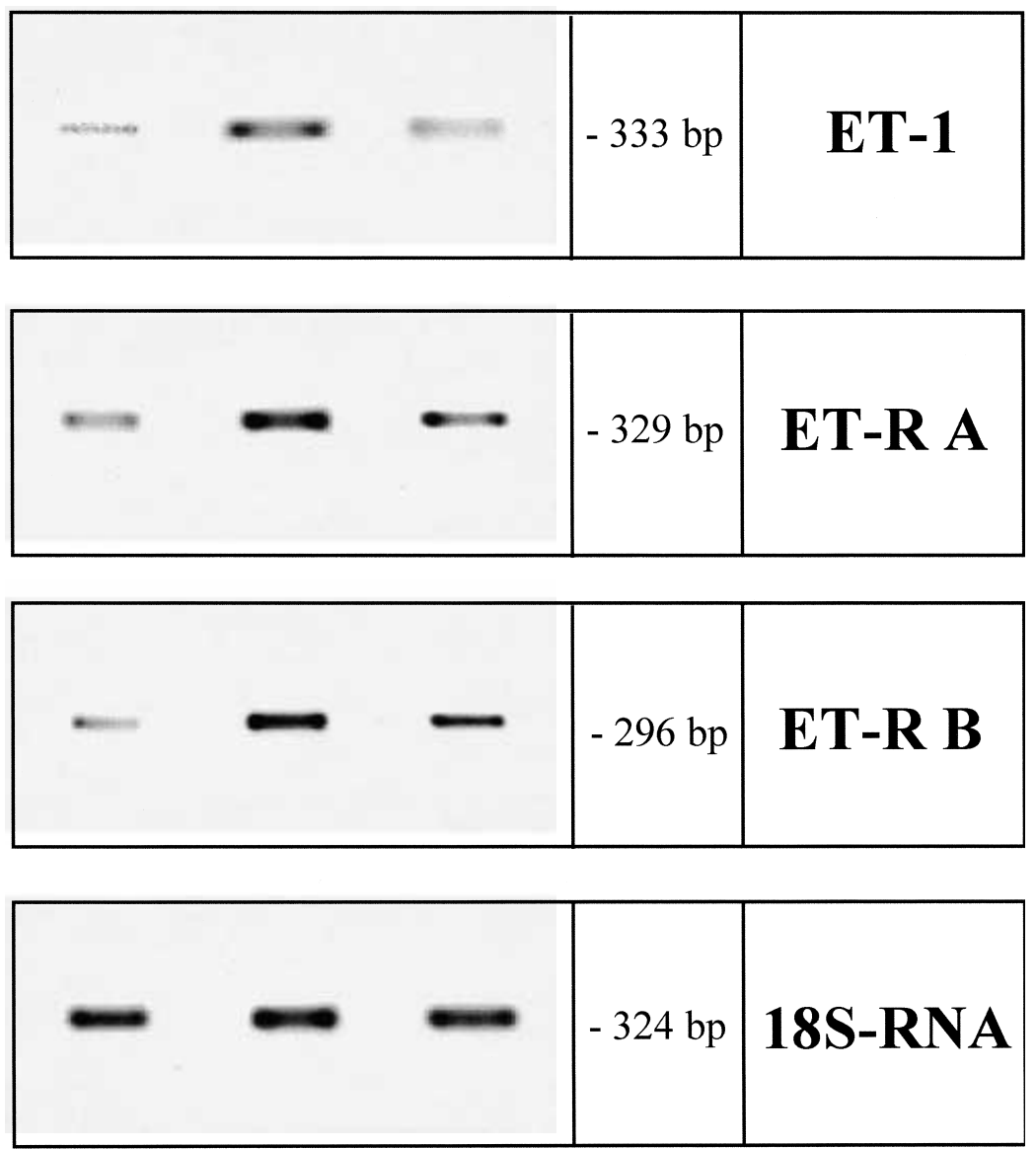

\section{Follicular Post Luteal ovulatory}

\section{Stage of the estrous cycle}

Figure 5 Specific RT-PCR products for ET-1 (333 bp), ET-R A (329 bp), ET-R B (296 bp) and $18 \mathrm{~S}$ rRNA (324 bp) separated by agarose gel electrophoresis. One representative result is shown for each estrous cycle phase $(n=4)$.

concentrations (Lundin et al. 1989). OT increased the contractile activities in the cow oviduct isthmus (Ruckebusch \& Bayard 1975, Bennett et al. 1988), and oviduct musculature was most sensitive to physiological concentrations of OT at estrus in the ewe (Gilbert et al. 1992). Furthermore, the ovine oviduct contains more OT receptors at the postovulatory phase than during the luteal phase (Ayad et al. 1991). These observations suggest that OT may play a role in the regulation of oviductal functions (Schaeffer et al. 1984), and stimulate oviduct motility involved with successful egg collection and/or sperm embryo transport, as postulated in the ewe (Wallace et al. 1991). However, we recently reported that the local distribution of OT in the bovine oviduct was low, and did not vary with the stage of the estrous cycle (Wijayagunawardane et al. 1998). The novel inhibitory effect of $\mathrm{OT}$ on the contractile mechanisms in the oviduct observed in the present study strongly suggests a possible inhibitory action of luteal OT on the LH-induced oviductal contraction through the suppression of the LHstimulated $\mathrm{PGE}_{2}, \mathrm{PGF}_{2 \alpha}$ and ET-1 secretion during the postovulatory period. Although the possible mechanisms involved in this phenomenon are unclear, the inhibitory effect of OT may be due to interaction of LH and OT at the receptor, COX or PK level. Further study is needed to clarify these points.

In conclusion, the overall results suggest that the preovulatory $\mathrm{LH}$ surge, together with increasing $\mathrm{E}_{2}$ secretion 
A) ET-1-PCR

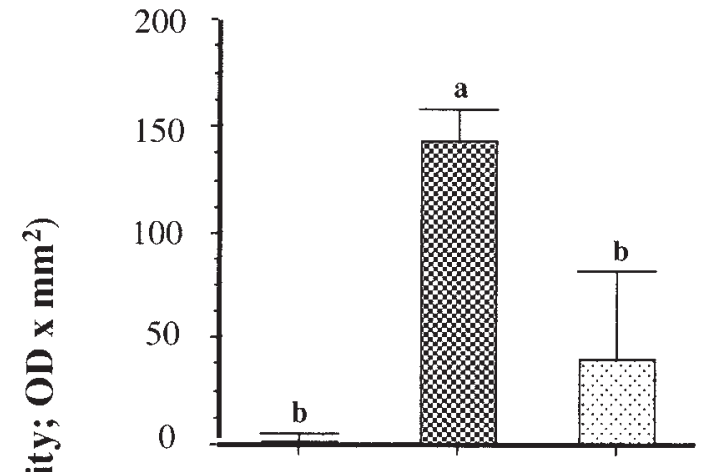

\section{B) ET-R A-PCR}

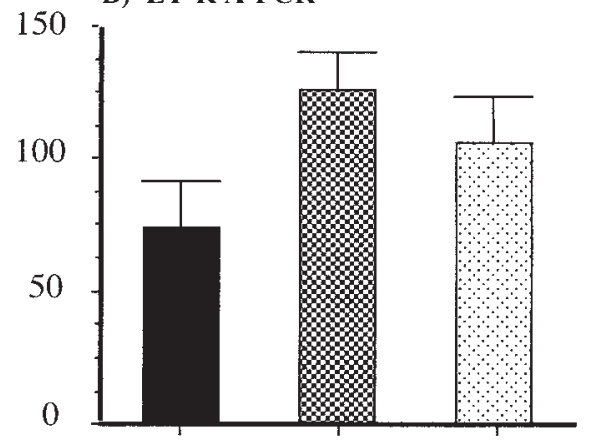

\section{C) ET-R B-PCR}

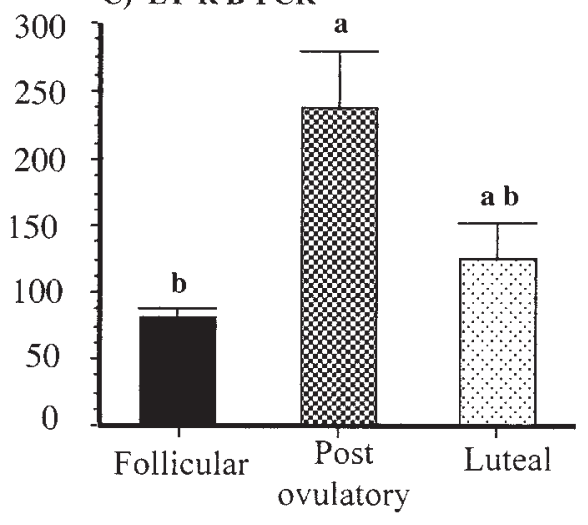

\section{Stage of the estrous cycle}

Figure 6 The relative amount of mRNA (integrated absorbance; means \pm S.E.M.) is depicted for each PCR result: (A) ET-1 PCR,

(B) ET-R A PCR and (C) ET-R B PCR $(n=4)$. Different letters above the bars denote significance at $P<0 \cdot 05$.

from the Graafian follicle and basal $\mathrm{P}_{4}$ levels from the regressing $\mathrm{CL}$, induce maximum stimulation of oviductal production of $\mathrm{PG}$ and $\mathrm{ET}-1$, resulting in an active oviduct contraction. Moreover, ET-1 may be a local mediator of LH modulation of oviduct PG synthesis. OT released from the newly formed CL appears to block these mechanisms, thus inhibiting the contraction to secure slow transport of the embryo to the uterus.

\section{Acknowledgements}

This study was supported in part by USDA grant No. 96-35203-3428. The authors wish to thank Dr D Schams, Technical University of Munich, Germany, for ET-1 antiserum, Dr S Ito, Kansai University of Medicine, Japan, for PG antiserum, Dr D J Bolt, USDA Animal Hormone program, for bovine LH, and Fresenius AG, St Wendal, Germany for the microdialysis capillary membranes.

\section{References}

Acosta TJ, Miyamoto A, Ozawa T, Wijayagunawardane MPB \& Sato K 1998 Local release of steroid hormones, prostaglandin $E_{2}$ and endothelin-1 from bovine mature follicles in vitro: effects of luteinizing hormone, endothelin-1 and cytokines. Biology of Reproduction 59 437-443.

Atanackovic D, Frkovic A, Mirosevic L \& Frkovic A 1983 The effect of prostaglandin $\mathrm{F}_{2 \alpha}$ and indomethacin on the longitudinal musculature of the human oviduct. Jugoslavian Ginekologiju $i$ Opstetriciju 23 112-115.

Ayad V, Guldenaar S \& Wathes D 1991 Characterization and localization of oxytocin receptors in the uterus and oviduct of the non-pregnant ewe using an iodinated receptor antagonist. Journal of Endocrinology 128 187-195.

Barber D, Sieck G, Fitzpatrick L \& Miller V 1996 Endothelin receptors are modulated in association with endogenous fluctuations in estrogen. American Journal of Physiology 271 H1999-2006.

Barbosa I, Maia HJ, Dourado V \& Coutinho E 1980 Effect of prostaglandin $\mathrm{F}_{2 \alpha}$ on oviduct contractility in marmoset monkeys. Fertility and Sterility 33 197-200.

Bennett W, Watts T, Blair W, Waldhalm S \& Fuquay J 1988 Patterns of oviducal motility in the cow during the oestrous cycle. Journal of Reproduction and Fertility 83 537-543.

Chomczynski P \& Sacchi N 1987 Single-step method of RNA isolation by acid guanidinium thiocyanate-phenol-chloroform extraction. Analytical Biochemistry 162 156-159.

Collett G, Kohnen G, Campbell S, Davenport A, Jeffers M \& Cameron I 1996 Localization of endothelin receptors in human uterus throughout the menstrual cycle. Molecular Human Reproduction 2 439-444.

Derecka K, Pietila E, Rajaniemi H \& Ziecik A 1995 Cycle dependent $\mathrm{LH} / \mathrm{hCG}$ receptor gene expression in porcine nongonadal reproductive tissues. Journal of Physiology and Pharmacology $\mathbf{4 6}$ $77-85$.

Emori T, Hirata Y, Ohta K, Kanno K, Eguchi S, Imai T, Shichiri M \& Marumo F 1991 Cellular mechanism of endothelin-1 release by angiotensin and vasopressin. Hypertension 18 165-170.

Gabler C, Lauer B, Einspanier A, Schams D \& Einspanier R 1997 Detection of mRNA and immunoreactive proteins for acidic and basic fibroblast growth factor and expression of the fibroblast growth factor receptors in the bovine oviduct. Journal of Reproduction and Fertility 109 213-221.

Gabler C, Plath-Gabler A, Einspanier A \& Einspanier R 1998 Insulin-like and fibroblast growth factors and their receptors are differentially expressed in the oviducts of the common marmoset monkey (Callithrix jacchus) during the ovulatory cycle. Biology of Reproduction 58 1451-1457. 
Gilbert C, Cripps P \& Wathes D 1992 Effect of oxytocin on the pattern of electromyographic activity in the oviduct and uterus of the ewe around oestrus. Reproduction, Fertility and Development 4 193-203.

Halbert S \& Conrad J 1975 In vitro contractile activity of the mesotubarium superius from the rabbit oviduct in various endocrine states. Fertility and Sterility 26 248-256.

Han S, Lei Z \& Rao C 1996 Up-regulation of cyclooxygenase-2 gene expression by chorionic gonadotropin in mucosal cells from human fallopian tubes. Endocrinology 137 2929-2937.

Ireland J, Murphee R \& Coulson P 1980 Accuracy of predicting stages of bovine estrous cycle by gross appearance of the corpus luteum. Journal of Dairy Science 63 155-160.

Kisielewska J, Flint A \& Ziecik A 1996 Phospholipase C and adenylate cyclase signalling systems in the action of hCG on porcine myometrial smooth muscle cells. Journal of Endocrinology 148 175-180.

Labadia A, Costa G, Jimenez E, Triguero D \& Garcia-Pascual A 1997 Endothelin receptor-mediated $\mathrm{Ca}^{2+}$ mobilization and contraction in bovine oviductal arteries: comparison with noradrenaline and potassium. General Pharmacology 29 611-619.

Lei Z, Toth P, Rao C \& Pridham D 1993 Novel coexpression of human chorionic gonadotropin (hCG)/human luteinizing hormone receptors and their ligand hCG in human fallopian tubes. Journal of Clinical Endocrinology and Metabolism 77 863-872.

Lindblom B \& Andersson A 1985 Influence of cyclooxygenase inhibitors and arachidonic acid on contractile activity of the human Fallopian tube. Biology of Reproduction 32 475-479.

Lindblom B, Ljung B \& Hamberger L 1979 Adrenergic and novel non-adrenergic neuronal mechanisms in the control of smooth muscle activity in the human oviduct. Acta Physiologica Scandinavica 106 215-220.

Lundin S, Forman A, Rechberger T, Svane D \& Andersson K 1989 Immunoreactive oxytocin and vasopressin in the non-pregnant human uterus and oviductal isthmus. Acta Endocrinologica 120 239-244.

Miyamoto A \& Schams D 1991 Oxytocin stimulates progesterone release from microdialyzed bovine corpus luteum in vitro. Biology of Reproduction 44 1163-1170.

Miyamoto A, Tashiro Y, Nakatsuka T, Meyer H, Taguchi K, Abe N \& Fukui Y 1995 Effect of tumor necrosis factor- $\alpha$ and interleukin-1 on local release of progesterone, prostaglandin $\mathrm{F}_{2 \alpha}$ and oxytocin in microdialyzed ovine corpus luteum in vitro. Assisted Reproductive Technology/Andrology 8 21-32.

Miyamoto A, Kobayashi S, Arata S, Ohtani M, Fukui Y \& Schams D 1997 Prostaglandin $\mathrm{F}_{2 \alpha}$ promotes the inhibitory action of endothelin-1 on the bovine luteal function in vitro. Journal of Endocrinology 152 R7-R11.

Orlicky D \& Williams-Skipp C 1993 Immunohistochemical localization of $\mathrm{PGF}_{2 \alpha}$ receptor in the rat oviduct. Prostaglandins, Leukotrienes and Essential Fatty Acids 48 185-192.

Rao C, Li X, Toth P, Lei Z \& Cook V 1993 Novel expression of functional human chorionic gonadotropin/luteinizing hormone receptor gene in human umbilical cords. Journal of Clinical Endocrinology and Metabolism 77 1706-1714.

Rodriguez-Martinez H, Einarsson S \& Larsson B 1982a Spontaneous motility of the oviduct in the anaesthetized pig. Journal of Reproduction and Fertility 66 615-624.
Rodriguez-Martinez H, Einarsson S, Larsson B, Akusu M \& Settergren I 1982b Spontaneous motility of the pig oviduct in vitro. Biology of Reproduction 26 98-104.

Rosselli M, Imthurn B, Macas E \& Keller P 1994 Endothelin production by bovine oviduct epithelial cells. Journal of Reproduction and Fertility 101 27-30.

Ruckebusch Y \& Bayard F 1975 Motility of the oviduct and uterus of the cow during the oestrous cycle. Journal of Reproduction and Fertility 43 23-32.

Schaeffer J, Liu J, Hsueh A \& Yen S 1984 Presence of oxytocin and arginine vasopressin in human ovary, oviduct, and follicular fluid. Journal of Clinical Endocrinology and Metabolism 59 970-973.

Schramm W, Einer-Jensen N, Schramm G \& McCracken J 1986 Local exchange of oxytocin from the ovarian vein to ovarian arteries in sheep. Biology of Reproduction 34 671-680.

Shemesh M, Gurevich M, Mizrachi D, Dombrovski L, Stram Y, Fields M \& Shore L 1997 Expression of functional luteinizing hormone $(\mathrm{LH})$ receptor and its messenger ribonucleic acid in bovine uterine veins: LH induction of cyclooxygenase and augmentation of prostaglandin production in bovine uterine veins. Endocrinology 138 4844-4851.

Spilman C \& Harper M 1975 Effects of prostaglandins on oviductal motility and egg transport. Gynecological Investigation $\mathbf{6}$ 186-205.

Sun T, Lei Z \& Rao C 1997 A novel regulation of the oviductal glycoprotein gene expression by luteinizing hormone in bovine tubal epithelial cells. Molecular and Cellular Endocrinology 131 97-108.

Toth P, Li X, Lei Z \& Rao C 1996 Expression of human chorionic gonadotropin (hCG)/luteinizing hormone receptors and regulation of the cyclooxygenase- 1 gene by exogenous hCG in human fetal membranes. Journal of Clinical Endocrinology and Metabolism $\mathbf{8 1}$ $1283-1288$

Tsunoda H, Miyauchi T, Fujita K, Kubo T \& Goto K 1993 Mechanism of rat uterine smooth muscle contraction induced by endothelin-1. British Journal of Pharmacology 110 1437-1446.

Wallace J, Helliwell R \& Morgan P 1991 Autoradiographical localization of oxytocin binding sites on ovine oviduct and uterus throughout the oestrous cycle. Reproduction, Fertility and Development 3 127-135.

Weber J, Freeman D, Vanderwall D \& Woods G 1991 Prostaglandin $\mathrm{E}_{2}$ hastens oviductal transport of equine embryos. Biology of Reproduction 45 544-546.

Wijayagunawardane MPB, Cerbito WA, Miyamoto A, Acosta TJ, Takagi M, Miyazawa K \& Sato K 1996 Oviductal progesterone concentration and its spatial distribution in cyclic and early pregnant cows. Theriogenology 46 1149-1158.

Wijayagunawardane MPB, Miyamoto A, Cerbito WA, Acosta TJ, Takagi M \& Sato K 1998 Local distributions of oviductal estradiol, progesterone, prostaglandins, oxytocin and endothelin-1 in the cyclic cow. Theriogenology 49 607-618.

Received 13 March 2000

Accepted 31 August 2000 